\title{
Prevalence of risk factors for noncommunicable diseases in an indigenous community in Santiago Atitlán, Guatemala
}

\author{
David Chen, ${ }^{1}$ Álvaro Rivera-Andrade, ${ }^{2}$ Jessica González, ${ }^{3}$ David Burt, ${ }^{4}$ \\ Carlos Mendoza-Montano, ${ }^{2}$ James Patrie, ${ }^{5}$ and Max Luna ${ }^{6}$
}

Suggested citation Chen D, Rivera-Andrade A, González J, Burt D, Mendoza-Montano C, Patrie J, Luna M. Prevalence of risk factors for noncommunicable diseases in an indigenous community in Santiago Atitlán, Guatemala. Rev Panam Salud Publica. 2017;41:e7.

Key words
Objective. To describe the prevalence of noncommunicable disease (NCD) risk factors and assess knowledge of those risk factors in the indigenous community of Santiago Atitlan in Guatemala, a lower-middle income country.

Methods. A population-based, cross-sectional study was conducted using a modified version of the World Health Organization's STEPS protocol. Adults aged 20-65 years were surveyed regarding demographics and NCD risk factors, and the survey was followed by anthropometric and biochemical measurements.

Results. Out of 501 screened individuals, 350 respondents were enrolled. The mean age was 36.7 years, and $72.3 \%$ were women. Over 90\% reported earning less than US\$ 65 per month. Almost $80 \%$ were stunted. Among women, $37.3 \%$ were obese and over three-quarters had central obesity. Over three-quarters of the entire group had dyslipidemia and 18.3\% had hypertension, but only $3.0 \%$ had diabetes. Overall, 36.0\% of participants met criteria for metabolic syndrome. There was no significant association between participants' education and NCD risk factors except for an inverse association with obesity by percent body fat.

Conclusions. Santiago Atitlán is a rural, indigenous Guatemalan community with high rates of poverty and stunting coexisting alongside high rates of obesity, particularly among women. Additionally, high rates of hypertension and dyslipidemia were found, but a low rate of diabetes mellitus. Knowledge of NCDs and their risk factors was low, suggesting that educational interventions may be a high-yield, low-cost approach to combating NCDs in this community.

Cardiovascular diseases; metabolic diseases; risk factors; obesity; indigenous population; Guatemala.
Public health efforts in developing countries have traditionally focused on infectious diseases and malnutrition. However, there is growing international

\footnotetext{
University of Virginia School of Medicine, Charlottesville, Virginia, United States of America.

Institute of Nutrition of Central America and Panama, Guatemala City, Guatemala.

3 University of Virginia-Guatemala Initiative, Quetzaltenango, Guatemala.

4 Department of Emergency Medicine, University of Virginia School of Medicine, Charlottesville, Virginia, United States of America.
}

recognition that the burden of chronic noncommunicable diseases (NCDs), such as diabetes and cardiovascular disease, poses a public health concern in

\footnotetext{
Department of Public Health Sciences, University of Virginia School of Medicine, Charlottesville, Virginia, United States of America.

Division of Cardiovascular Medicine, University of Virginia School of Medicine, Charlottesville, Virginia, United States of America. Send correspondence to: Max Luna, mal4pe@virginia.edu
}

lower-middle income countries (LMICs) (1-2). Globally, over $80 \%$ of NCD deaths now occur in LMICs (3). In the Latin America and Caribbean region, NCDs are estimated to be responsible for over half of the disability-adjusted life years lost due to illness (4). Like many other LMICs, Guatemala is undergoing an epidemiologic transition from the predominance of infectious diseases toward the ascendancy of chronic NCDs, largely driven by population aging (5-6), 
changes in diet, and reductions in physical activity. Previous reports suggest that Guatemala is in an intermediate stage of the transition, where childhood malnutrition and infectious diseases coexist with increasing rates of obesity-related conditions (7-8). In Guatemala, the percentage of deaths attributed to cardiovascular disease (CVD) has increased in recent decades from $7 \%$ in 1986 to $17 \%$ in 2009, when it represented the second most common cause of death in the country (9). As communities in Guatemala continue to move away from traditional subsistence farming-based lifestyles, diets are transitioning toward the atherogenic and diabetogenic diets seen in most developed countries. As this transition continues, it is expected that NCDs and their risk factors will increase in indigenous communities such as Santiago Atitlán (10).

Santiago is a mixed rural and semi-urban indigenous community in Sololá Department, Guatemala, with a population of approximately 44000 (11). The majority of the inhabitants speak Tzu'tujil as their native language and Spanish as a second language. Santiago has the highest rate of rural poverty and the lowest literacy rate in the department of Sololá (12), which underscores the importance of assessing NCD knowledge. There is a dearth of quality data on health metrics in Santiago and other indigenous communities, but early reports using convenience samples suggest that NCDs such as hypertension and diabetes are underdiagnosed and undertreated in this rural Mayan community (13-14). Therefore, it is important to study these diseases and their risk factors systematically.

The primary objective of this study was to describe the burden of NCD risk factors in Santiago Atitlán and a secondary objective was to assess community knowledge about NCDs, with the study population in Santiago Atitlán serving as a sample of the indigenous population in Guatemala. Our hypothesis was that this study would confirm the increasing prevalence of NCD risk factors suggested in earlier reports (13-14).

\section{MATERIALS AND METHODS}

\section{Participants}

The Noncommunicable Disease Surveillance (NCDS) Study was designed as a cross-sectional study in a simple random sample to examine the prevalence of behavioral and biochemical NCD risk factors using a modified Spanish version of the World Health Organization's (WHO) STEPS protocol (15). Application of the Spanish study instruments frequently required verbal translation to Tzu'tujil at the time of the interview since Tzu'tujil is not a written language.

The population of approximately 44000 was distributed in 20 cantones or districts with a total of 7559 households and an average of 5.8 individuals per household. In order to achieve a geographically representative sample of the population, the number of households to be approached in each cantón was determined in proportion to the estimated adult population according to the latest available census data (16). Study households were randomly preselected using household level maps. From October 2012 to April 2013, 501 eligible individuals $(1.1 \%$ of the total population) were screened for participation in the study. Adults aged 20-65 years who were living in Santiago and were able to provide informed consent were eligible. If a household contained more than one eligible participant, one was randomly selected. Sampling weights were generated to reflect the probability of selection at each stage.

\section{Study tools}

The WHO STEPS protocol is a standardized tool designed for assessing NCD risk factors in developing countries. The original STEPS protocol was modified to be culturally appropriate, to include measurement of body fat percentage (BFP), and to add nine multiple choice questions pertaining to basic knowledge of NCDs (survey instrument available upon request). A pilot phase was conducted in which the study tools were administered to small focus groups drawn from the community to identify potential areas of confusion and to enhance cultural appropriateness. The STEPS protocol is described in detail elsewhere (15). In brief, our instruments included the following:

Step 1: Sociodemographic and behavioral variables. A structured interview based on a standardized questionnaire was used to collect demographic information and data on risk factors. Topics included diet, exercise, smoking, alcohol use, family history, and personal history of NCDs.

Step 2: Anthropometric measurements. Height, weight, waist circumference, BFP, and blood pressure were measured using standardized instruments and protocols. After the subject had been at rest for 5 minutes, blood pressure and heart rate were measured in the sitting position three times at 3-5 minute intervals with the appropriate size automated blood pressure cuff (Omron 5 series). Reported blood pressure is the mean of the two closest readings of both systolic and diastolic pressures. Waist circumference was measured one inch above the umbilicus. Bioelectrical impedance was used to measure BFP according to the protocol of the medical device (Bodystat 1500) (17).

Step 3: Biochemical measurements. Finger-stick blood samples were obtained by a trained physician after a minimum 12-hour fast. Laboratory measurements of serum glucose and lipids were performed using the Cholestech LDX following the manufacturer's procedure protocol. This finger-stick technique has been validated against venous blood sampling (18).

\section{Interviewers}

During the design of the NCDS, an emphasis was placed on building local capacity. In furtherance of this philosophy, nine local, bilingual (Spanish and Tzu'tujil) community health workers were recruited and trained in the use of study protocols and also participated in a customized training and certification program in human subject research ethics.

\section{NCD risk factors}

Hypertension was defined as systolic pressure $\geq 140 \mathrm{mmHg}$, diastolic pressure $\geq 90 \mathrm{mmHg}$, or being on medical therapy for hypertension. Overweight was defined as body mass index (BMI) of 2529.9 , and obese as BMI $\geq 30.0$. By BFP, obesity was defined as $\geq 25 \%$ for men and $\geq 35 \%$ for women (19). Central obesity was waist circumference (WC) $\geq 80$ $\mathrm{cm}$ for women and $\mathrm{WC} \geq 90 \mathrm{~cm}$ for men, in accordance with the International 
Diabetes Federation (IDF) definition for Latinos. Stunting was defined as a height of $<162 \mathrm{~cm}$ for men and $<150 \mathrm{~cm}$ for women, which corresponds to two standard deviations below the median of the 2000 reference population in the United States of America (20). Diabetes was defined as fasting glucose $\geq 126$ $\mathrm{mg} / \mathrm{dL}$ or taking medication for known diabetes. Hypercholesterolemia was total cholesterol $\geq 200 \mathrm{mg} / \mathrm{dL}$, hypertriglyceridemia was triglycerides $\geq 150$ $\mathrm{mg} / \mathrm{dL}$, and low high-density lipoprotein (HDL) was HDL $\leq 40 \mathrm{mg} / \mathrm{dL}$ for men or HDL $\leq 50 \mathrm{mg} / \mathrm{dL}$ for women; being on medication to treat these conditions also met the definition. Metabolic syndrome was identified according to the IDF criteria (21). Ten-year risk for cardiovascular events was calculated using WHO risk charts (22).

Smoking and alcohol use were defined as any current use. Participants were considered sedentary if they reported less than 60 minutes of moderate to intense physical activity per week. Adequate fruit/vegetable intake was defined as at least five servings of fruits and vegetables per day.

\section{Data management and statistical analysis}

Data entry was done using EpiData 2.1 and was subjected to systematic review for outlying data following the WHO STEPS protocol. Statistical analysis was conducted with SAS 9.4 (SAS Institute Inc., Cary, North Carolina, United States of America).

All survey responses were analyzed in accordance with the single-stage stratified random sampling design. The Santiago Atitlán community served as the stratification variable. Population and subpopulation parameter estimates for prevalence and averages were derived based on sampling inclusion probabilities (23) in which survey weights assigned to the respondents corresponded to age and gender demographic information from the 2005 Santiago Atitlán census. Variability in the population or subpopulation parameter estimates was ascertained via the Taylor series linear expansion technique (24).

Gender comparisons of continuous variables were conducted via linear regression weighted by probability of inclusion in the single-stage stratified random sample, while risk factor prevalences were compared between female and male subpopulations via similarly probability -weighted logistic regression. Association of NCD risk factors to literacy and education level was assessed via probability -weighted logistic regression. In these analyses, the NCD risk factors were treated as the response variables, while literacy and education were the predictor variables in age- and gender-adjusted analyses. Literacy was modeled as a binary variable and levels of education were modeled as ordinal categories. The Bonferroni correction was applied to correct for multiple comparisons.

\section{Ethical considerations}

The study protocol conforms to the ethical guidelines of the 1975 Declaration of Helsinki and was approved by both partner institutions' ethics committees: the University of Virginia's Human Subjects' Research Institutional Review Board (IRB) (Charlottesville, Virginia, United States of America) and the Institute of Nutrition of Central America and Panama (INCAP) IRB (Guatemala City, Guatemala). All participants gave verbal and written informed consent. All collected data were anonymized prior to analysis using linked participant identification numbers. Identifiable information was kept under lock and key at INCAP's central office and was accessible only to the authors of the study.

\section{RESULTS}

\section{Participants}

Of the 501 persons screened, 350 agreed to participate, a response rate of $70.0 \%$. Among these 350 participants, 308 subjects $(88.0 \%)$ provided blood for biochemical analysis. Participants who provided blood did not differ from those who did not with respect to age, sex, or literacy (as a surrogate of socioeconomic status).

The mean age of the participants was 36.7 years old, and $72.3 \%$ were women. Educational attainment was low; $45.3 \%$ (95\% confidence interval (CI): 38.9$51.8 \%$ ) had no formal education, and an additional $23.7 \%$ (95\% CI: $13.4-28.9 \%$ ) did not complete primary school. Literacy was low at $54.2 \%$ (95\% CI: 47.6$60.8 \%$ ). Most men (83.1\%; 95\% CI: 72.3-93.0\%) were self-employed, while most women $(75.4 \%$; $95 \%$ CI: $69.0-81.7 \%)$ were homemakers. Most participants (86.2\%; 95\% CI: 80.5-91.9\%) reported earning less than 500 quetzales per month ( US\$ 65) (Table 1).

With respect to behavioral risk factors, prevalence of current smoking was 13.0\% (95\% CI: 5.4-20.6\%) among men, while no women reported tobacco use. Similarly, no women reported alcohol use, but $19.6 \%$ (95\% CI: $10.6 \%-28.6 \%$ ) of men did. Most participants (75.0\%; 95\% CI: $68.7-81.2 \%$ ) reported being sedentary, with $<60$ minutes of physical activity per week. The majority (91.6\%; 95\% CI: 88.4$94.8 \%$ ) reported consuming less than the minimum recommended intake of fruits and vegetables (Table 2).

Anthropometric measures were notable for a high prevalence of stunting: 79.8\% (95\% CI: $73.8-85.8 \%$ ). There was also a high rate of overweight and obesity, with a mean BMI of 27.0 (Table 3). Over one-third (36.7\%; 95\% CI: 29.5$42.6 \%)$ of participants were overweight (BMI 25-29.9), and an additional 25.2\% (95\% CI: 19.5-30.9\%) were obese (BMI $\geq 30$ ). This high rate of obesity was consistent with other measures of adiposity, including obesity by BFP (55.3\%; 95\% CI: $48.0-62.5 \%$ ) and central obesity (45.1\%; 95\% CI: 38.1-52.1\%) (Table 2). The gender difference with respect to obesity was large and statistically significant, with women far more likely to be obese than men as measured by BMI (37.3\% vs. $12.4 \%$, respectively; $P<0.05$ ). This pattern held true for obesity by WC and BFP.

Hypertension was prevalent at $18.3 \%$ (95\% CI: $13.1-23.6 \%$ ), and rates did not differ significantly between the sexes (Table 2). Among hypertensive participants, the diagnosis was new to over three-quarters of them.

With respect to biochemical measurements, hypertriglyceridemia was highly prevalent at $64.0 \%(95 \% \mathrm{CI}$ : $56.9-71.1 \%$ ), while only $11.9 \%$ (95\% CI: $7.4-16.5 \%$ ) had a total cholesterol level higher than $200 \mathrm{mg} / \mathrm{dL}$. Most of the group had low HDL levels $(82.7 \%)$. None of the dyslipidemic patients had previously been diagnosed. In contrast, the rate of diabetes (fasting glucose $\geq 126 \mathrm{mg} / \mathrm{dL}$ ) was only $3.0 \%$ (95\% CI: $1.1-4.8 \%$ ). Of those with diabetes, less than half knew they had elevated glucose. There was no statistically significant difference in the prevalence of any of the biochemical NCD risk factors between the sexes. 
TABLE 1. Demographic characteristics, by sex, in the Noncommunicable Disease Surveillance (NCDS) study population in Santiago Atitlán, Guatemala, 2012-2013

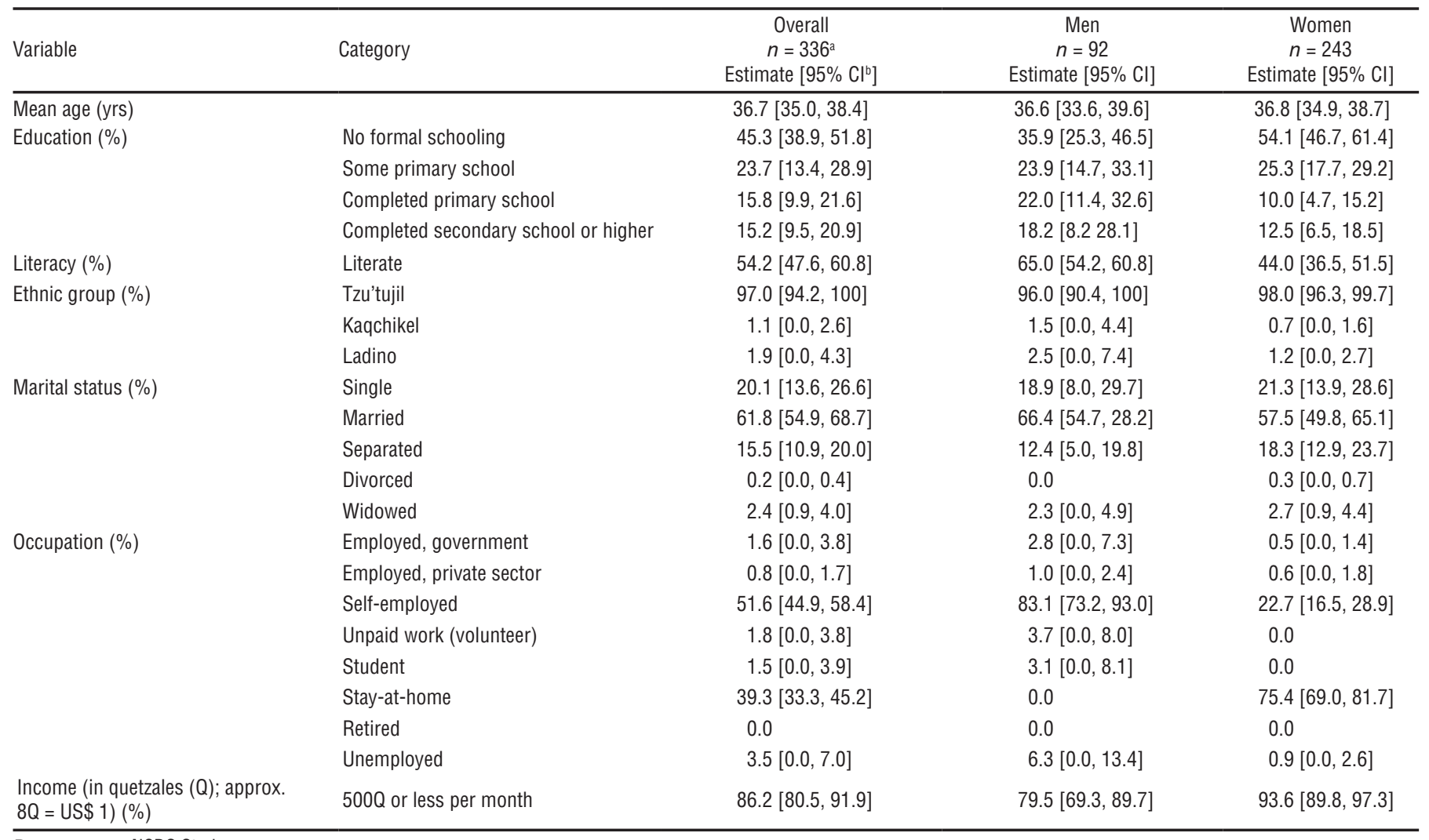

Data source: NCDS Study.

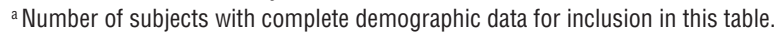

${ }^{\mathrm{b}} \mathrm{Cl}=$ confidence interval.

The prevalence of metabolic syndrome was $36.0 \%$ (95\% CI: $29.2-42.8 \%)$; it was more common in women $(56.7 \% ; 95 \% \mathrm{CI}$ : 48.4-65.0\%) than in men $(14.7 \%$; 95\% CI: $6.2-23.3 \%)(P<0.05)$. Despite this finding, most of the population was classified as having a low 10-year risk of a major cardiovascular event, with only $2.8 \%$ of individuals over age 40 classified as intermediate-to-high risk (the WHO risk score tables only apply to people above 40 years of age).

There was a strong inverse association between obesity by BFP and both literacy and level of education. The odd ratios were 0.33 (95\% CI: $0.18-0.64, P<0.05)$ and 0.52 (95\% CI: $0.37-0.72, P<0.001)$, respectively. In other words, the more highly educated the individual, the lower the likelihood that he or she was obese. No other statistically significant associations were found between literacy or level of education and NCD risk factors (Table 4).

Participant knowledge of NCDs was low, with only one-fifth of participants
(20.1\%) answering at least seven of nine simple questions about NCDs correctly (Table 5).

\section{DISCUSSION}

This study joins a small body of research examining noncommunicable disease epidemiology in indigenous Latin American populations. It identified a surprisingly high prevalence of numerous risk factors, including obesity in women, hypertension, hypertriglyceridemia, and metabolic syndrome.

The prevalence of obesity of $37.3 \%$ among women in this study was much higher than the $25.8 \%$ found in a cohort of nonindigenous, urban Guatemalans living in Villa Nueva (25), and it approaches the $43 \%$ obesity rate among Latino women living in the United States of America (26). On the other hand, men in this study were less obese $(12.4 \%)$ than both those in Villa Nueva $(16.0 \%)$ and the United States cohort (37\%). The prevalence of central obesity was even more striking than obesity by BMI. Over three-quarters of the women in this study had central obesity, which is higher than the $68.3 \%$ prevalence among women in Villa Nueva. This is a particularly notable addition to the literature, since it was expected that women in a rural community such as Santiago would have lower rates of obesity than their urban counterparts. This study was not designed to be able to address directly the question of why women's rates of obesity were so much higher than men's, but the explanation may relate to the differing lifestyles of men and women in Santiago. Men typically engage in physically intensive labor, while women are more likely to work at home, in markets, or in other occupations requiring less physical activity. Alternatively, it is possible that genetic influences predispose these women to central obesity. Further research is needed to explore these mechanisms and to determine whether the high rate of obesity increases cardiovascular and diabetes risk, as it does in other ethnic groups. 
TABLE 2. Prevalence and $95 \%$ confidence intervals (CI) of anthropometric and biochemical noncommunicable disease risk factors, by sex, in the Noncommunicable Disease Surveillance study population in Santiago Atitlán, Guatemala, 2012-2013

\begin{tabular}{|c|c|c|c|c|}
\hline \multicolumn{2}{|c|}{ Variable } & $\begin{array}{l}\text { Overall }\left(n=296^{\mathrm{a}}\right) \\
\quad \%[95 \% \mathrm{Cl}]\end{array}$ & $\begin{array}{c}\text { Men }(n=83) \\
\%[95 \% \mathrm{Cl}]\end{array}$ & $\begin{array}{c}\text { Women }(n=213) \\
\%[95 \% \mathrm{Cl}]\end{array}$ \\
\hline \multicolumn{5}{|c|}{ Anthropometric risk factors ${ }^{b}$} \\
\hline \multirow[t]{3}{*}{ Body mass index (BMI) } & Normalc & $38.6[31.2,46.0]$ & $57.5[45.5,69.8]$ & $20.8[14.5,27.1]$ \\
\hline & Overweight ${ }^{c}$ & $36.7[29.5,42.6]$ & $30.1[19.1,41.1]$ & $41.9[34.2,49.6]$ \\
\hline & Obese $^{c}$ & $25.2[19.5,30.9]$ & $12.4[5.3,19.6]$ & $37.3[29.8,44.8]$ \\
\hline \multicolumn{2}{|l|}{ Central obesityc } & $45.1[38.1,52.1]$ & $14.8[6.5,23.1]$ & $73.6[66.3,80.3]$ \\
\hline \multicolumn{2}{|c|}{ Obesity by percentage body fat (BFP)c } & $55.3[48.0,62.5]$ & $35.7[24.5,46.9]$ & $73.8[66.5,81.0]$ \\
\hline \multicolumn{2}{|l|}{ Stunted } & $79.8[73.8,85.8]$ & $76.7[66.1,87.3]$ & $82.7[76.9,88.6]$ \\
\hline \multicolumn{2}{|l|}{ Hypertension } & $18.3[13.1,23.6]$ & $20.0[10.9,29.2]$ & $16.8[11.5,22.1]$ \\
\hline \multicolumn{2}{|l|}{ Known hypertension } & $3.3[0.64,6.1]$ & $4.3[0.0,9.5]$ & $2.4[0.5,4.4]$ \\
\hline \multicolumn{2}{|c|}{ Previously unknown hypertension } & $15.0[10.3,19.7]$ & $15.7[7.6,23.9]$ & $14.3[9.3,19.4]$ \\
\hline \multicolumn{5}{|l|}{ Biochemical risk factors ${ }^{b}$} \\
\hline \multicolumn{2}{|l|}{ Diabetes mellitus (DM) } & $3.0[1.1,4.8]$ & $1.3[0.0,3.2]$ & $4.6[1.6,7.7]$ \\
\hline \multicolumn{2}{|l|}{ Known DM } & $1.3[0.1,2.5]$ & $1.3[0.0,3.2]$ & $1.4[0.0,2.9]$ \\
\hline \multicolumn{2}{|l|}{ Unknown DM } & $1.7[0.3,3.0]$ & & $3.2[0.5,5.9]$ \\
\hline \multicolumn{2}{|l|}{ Hypercholesterolemia } & $11.9[7.4,16.5]$ & $9.7[2.6,16.8]$ & $14.1[8.4,19.7]$ \\
\hline \multicolumn{2}{|l|}{ Low HDL cholesterol } & $82.7[76.9,88.5]$ & $78.0[67.5,88.5]$ & $87.2[81.8,92.7]$ \\
\hline \multicolumn{2}{|l|}{ Elevated LDL } & $5.0[2.2,7.8]$ & $3.9[0.0,7.8]$ & $6.0[2.0,9.9]$ \\
\hline \multicolumn{2}{|l|}{ Hypertriglyceridemia } & $64.0[56.9,71.1]$ & $65.1[53.0,77.2]$ & $62.9[55.3,70.5]$ \\
\hline \multicolumn{2}{|l|}{ Metabolic syndrome ${ }^{\mathrm{c}}$} & $36.0[29.2,42.8]$ & $14.7[6.2,23.3]$ & $56.7[48.4,65.0]$ \\
\hline \multicolumn{5}{|l|}{ Behavioral risk factors } \\
\hline \multicolumn{2}{|l|}{ Current tobacco use } & $6.3[2.5,10.0]$ & $13.0[5.4,20.6]$ & 0.0 \\
\hline \multicolumn{2}{|c|}{ Alcohol within last 12 months } & $9.4[4.9,13.9]$ & $19.6[10.6,28.6]$ & 0.0 \\
\hline \multicolumn{2}{|c|}{$<5$ Servings of fruits and vegetables per day } & $91.6[88.4,94.8]$ & $95.0[90.5,99.5]$ & $88.4[83.7,93.0]$ \\
\hline \multicolumn{2}{|l|}{ Sedentaryc } & $75.0[68.7,81.2]$ & $64.5[53.3,75.7]$ & $84.7[79.2,90.3]$ \\
\hline
\end{tabular}

Data Source: NCDS Study.

${ }^{a}$ Number of subjects with complete anthropometric and biochemical data.

${ }^{\circ}$ Overweight: BMI 25-29.9; Obese: BMI $\geq 30$; Central obesity: Waist circumference $\geq 90 \mathrm{~cm}$ in men or $\geq 80 \mathrm{~cm}$ in women; Obesity by BFP. $\geq 25 \%$ body fat in men or $\geq 35 \%$ body fat in women; Stunted: Height $\leq 162 \mathrm{~cm}$ in men or $\leq 150 \mathrm{~cm}$ in women; Hypertension: Systolic $\geq 140$ $\mathrm{mmHg}$ or diastolic $\geq 90 \mathrm{mmHg}$; Diabetes: Fasting glucose $\geq 126 \mathrm{mg} / \mathrm{dL}$; Hypercholesterolemia: Total cholesterol $\geq 200 \mathrm{mg} / \mathrm{dL}$; Low HDL: $\mathrm{HDL} \leq 40 \mathrm{mg} / \mathrm{dL}$ in men or $\leq 50 \mathrm{mg} / \mathrm{dL}$ in women; High LDL: LDL $\geq 130 \mathrm{mg} / \mathrm{dL}$; Hypertriglyceridemia: Triglycerides $\geq 150 \mathrm{mg} / \mathrm{dL}$; Metabolic syndrome by IDF criteria; Sedentary: $<60$ min of moderate-intense physical activity per week.

$P<0.05$ for difference between men and women by logistic regression.

Obesity was not the only NCD risk factor found to be markedly higher than expected. Over three-quarters of the study participants were dyslipidemic. However, unlike for obesity, there was no significant difference in prevalence between the sexes. Once again, the Santiago population was found to have a higher rate of dyslipidemias than their urban, nonindigenous counterparts in Villa Nueva. For instance, hypertriglyceridemia was present in $53.2 \%$ of Villa Nueva participants (25) compared to $64.0 \%$ in our indigenous population. Of interest is the difference in triglyceride levels between our study population and Latinos in the United States of America. Mean triglycerides in women and men were 209.7 $\mathrm{mg} / \mathrm{dL}$ and $221.5 \mathrm{mg} / \mathrm{dL}$, respectively, in this study versus $119.9 \mathrm{mg} / \mathrm{dL}$ and $149.0 \mathrm{mg} / \mathrm{dL}$, respectively, among U.S.
Latinos (27). Possible explanations of this high rate of hypertriglyceridemia include genetic predisposition and high intake of carbohydrates, including refined carbohydrates. More research is needed to explore the reasons for these differences.

The finding that the prevalence of major NCD risk factors in this rural indigenous community in a LMIC approaches that in the United States of America is a cause for concern and represents a public health threat for Guatemala. Indigenous communities constitute $40 \%$ of the Guatemalan population and are the most impoverished and underserved groups in the country. Thus, systematic and aggressive prevention efforts are needed alongside initiatives to increase availability of therapies for established NCDs.
The $18.3 \%$ prevalence of hypertension in this study's participants was similar to that reported in their urban, nonindigenous Guatemalan counterparts in Villa Nueva (17\%) but less than the $24.4 \%$ hypertension prevalence found among U.S. Latinos. It was surprising to find such a low rate of diabetes in our study, given the high degree of overweight and obesity. The prevalence of diabetes is also discordant with the prevalence of other NCD risk factors (25-27). One possible explanation is that this study may have captured the population at a very early stage in the epidemiologic transition. A small community-based study of 49 women from Santiago Atitlán in 2007 (28) found rates of overweight and obesity of $22.4 \%$ and $24.5 \%$, respectively. Those rates are notably lower than the ones found in this study five to six years later, although different sampling methods make direct comparison difficult. Nevertheless, the difference suggests the possibility that the prevalence of obesity has increased rapidly over the last decade and supports the theory that insufficient time has passed to see the metabolic effect of obesity leading to diabetes. An alternative explanation that merits consideration is that standard cut-offs for obesity and central obesity derived from non-Mayan populations may not accurately predict risk in this indigenous group. It has been well established that optimal BMI cut points can vary by population (29). For instance, there is variation in suggested BMI cut points among and between Asian populations (30), with obesity thresholds ranging from 26 to 31 . One study examined BMI and WC risk thresholds among nonindigenous Guatemalan adults and suggested that optimal risk category cut-offs may differ from international norms (20). Determination of the optimal BMI and WC cutoffs for predicting NCD risk in this and other ethnic groups remains an important area for future research.

No significant association was found between literacy or level of education and 15 out of 16 NCD risk factors after correcting for multiple comparisons (Table 4). This lack of consistent association between these two surrogates of socioeconomic status and NCD risk factors is consistent with other studies in LMICs (31). Poor communities in early epidemiologic transition exhibit heterogeneous 
TABLE 3. Values for anthropometric and biochemical risk factors and $95 \%$ confidence intervals (Cl), by sex, in the Noncommunicable Disease Surveillance (NCDS) study population in Santiago Atitlán, Guatemala, 2012-2013

\begin{tabular}{|c|c|c|c|c|}
\hline Variable & Units & $\begin{array}{c}\text { Overall } \\
n=296^{\mathrm{a}} \\
\text { Mean }[95 \% \mathrm{Cl}]\end{array}$ & $\begin{array}{c}\text { Men } \\
n=83 \\
\text { Mean }[95 \% \mathrm{Cl}]\end{array}$ & $\begin{array}{c}\text { Women } \\
n=213 \\
\text { Mean }[95 \% \mathrm{Cl}]\end{array}$ \\
\hline \multicolumn{5}{|l|}{ Anthropometric risk factors } \\
\hline $\mathrm{BMI}^{b}$ & & $27.0[26.3,27.6]$ & $25.3[24.3,26.3]$ & $28.5[27.9,29.2]$ \\
\hline Waist circumference ${ }^{b}$ & $\mathrm{~cm}$ & $83.7[82.4,85.1]$ & $81.7[79.1,83.7]$ & $85.6[83.9,87.4]$ \\
\hline Body fat percentage ${ }^{b}$ & $\%$ & $30.8[29.4,32.3]$ & $22.4[21.1,23.8]$ & $38.8[37.8,39.8]$ \\
\hline Height $^{b}$ & $\mathrm{~cm}$ & $151.5[150.2,152.8]$ & $157.9[156.1,159.7]$ & $145.4[144.5,146.3]$ \\
\hline Blood pressure, systolic & $\mathrm{mmHg}$ & $122.2[120.0,124.5]$ & $123.1[119.3,127.0]$ & $121.4[118.9,123.9]$ \\
\hline Blood pressure, diastolic & $\mathrm{mmHg}$ & $75.6[74.2,77.1]$ & $76.1[73.7,78.5]$ & $75.2[73.4,74.0]$ \\
\hline \multicolumn{5}{|l|}{ Biochemical risk factors } \\
\hline Total cholesterol & $\mathrm{mg} / \mathrm{dL}$ & $162.8[158.1,167.5]$ & $160.4[152.8,168.0]$ & $165.0[159.6,170.5]$ \\
\hline Triglycerides & $\mathrm{mg} / \mathrm{dL}$ & $215.4[197.9,232.9]$ & $221.5[194.0,249.0]$ & $209.7[187.8,231.5]$ \\
\hline HDL & $\mathrm{mg} / \mathrm{dL}$ & $36.5[34.5,38.6]$ & $34.9[31.3,38.5]$ & $38.1[36.0,40.1]$ \\
\hline LDL & $\mathrm{mg} / \mathrm{dL}$ & $87.6[84.2,91.0]$ & $89.0[83.9,94.0]$ & $86.3[81.6,91.0]$ \\
\hline
\end{tabular}

Data source: NCDS Study.

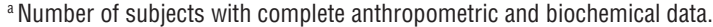

${ }^{\mathrm{b}} P<0.05$ for difference between men and women by linear regression.

TABLE 4. Odds ratios and $95 \%$ confidence intervals $(\mathrm{Cl})$ of association between noncommunicable disease risk factors and literacy or level of education, sex-adjusted, in the Noncommunicable Disease Surveillance (NDCS) study population in Santiago Atitlán, Guatemala, 2012-2013

\begin{tabular}{|c|c|c|c|c|}
\hline \multirow[b]{2}{*}{ Risk factor } & \multicolumn{2}{|c|}{$\begin{array}{c}\text { Literate } \\
\text { (Yes : No) }\end{array}$} & \multicolumn{2}{|c|}{$\begin{array}{c}\text { Level of education } \\
\text { (One category increase in education })^{b}\end{array}$} \\
\hline & $\begin{array}{l}\text { Adjusted odds } \\
\text { ratio }[95 \% \mathrm{Cl}]\end{array}$ & $\begin{array}{c}P \text {-value } \\
\text { (uncorrected } P \text { ) }\end{array}$ & $\begin{array}{l}\text { Adjusted odds ratio } \\
{[95 \% \mathrm{Cl}]}\end{array}$ & $\begin{array}{c}P \text {-value } \\
\text { (uncorrected } P \text { ) }\end{array}$ \\
\hline $\mathrm{BMI} \geq 25$ & $0.80[0.41,1.55]$ & $1.000(0.509)$ & $0.75[0.56,1.00]$ & $1.000(0.345)$ \\
\hline $\mathrm{BMI} \geq 30$ & $0.74[0.40,1.38]$ & $1.000(0.345)$ & $0.83[0.62,1.11]$ & $1.000(0.204)$ \\
\hline Central obesity & $0.66[0.33,1.34]$ & $1.000(0.252)$ & $0.75[0.54,1.03]$ & $1.000(0.074)$ \\
\hline $\begin{array}{l}\text { Obesity by body } \\
\text { fat percentage }\end{array}$ & $0.33[0.18,0.64]$ & $0.016(0.001)$ & $0.52[0.37,0.72]$ & $<0.001(<0.001)$ \\
\hline Stunted & $0.82[028,1.37]$ & $1.000(0.239)$ & $0.82[0.57,1.21]$ & $1.000(0.331)$ \\
\hline Hypertension & $0.67[0.37,1.20]$ & $1.000(0.177)$ & $0.72[0.52,0.99]$ & $0.688(0.043)$ \\
\hline Diabetes mellitus & $0.26[0.07,1.00]$ & $0.801(0.050)$ & $0.43[0.16,1.14]$ & $1.000(0.090)$ \\
\hline Hypercholesterolemia & $0.72[0.30,1.74]$ & $1.000(0.463)$ & $0.95[0.64,1.41]$ & $1.000(0.808)$ \\
\hline Low HDL cholesterol & $0.93[0.40,2.17]$ & $1.000(0.817)$ & $0.87[0.59,1.28]$ & $1.000(0.479)$ \\
\hline Elevated LDL & $0.34[0.10,1.10]$ & $1.000(0.073)$ & $0.78[0.42,1.46]$ & $1.000(0.437)$ \\
\hline Hypertriglyceridemia & $0.61[0.32,1.15]$ & $1.000(0.126)$ & $0.65[0.48,0.87]$ & $0.064(0.004)$ \\
\hline Metabolic syndrome & $0.68[0.35,1.33]$ & $1.000(0.265)$ & $0.72[0.53,0.99]$ & $0.720(0.045)$ \\
\hline Tobacco use ${ }^{c}$ & $0.31[0.07,1.30]$ & $1.000(0.109)$ & $0.31[0.14,0.74]$ & $0.128(0.008)$ \\
\hline Alcohol use in last 12 months $^{c}$ & $1.17[0.41,3.35]$ & $1.000(0.774)$ & $0.70[0.43,1.12]$ & $1.000(0.137)$ \\
\hline$<5$ Servings of fruits and vegetables per day & $1.48[0.63,3.52]$ & $1.000(0.370)$ & $1.53[0.61,3.86]$ & $1.000(0.362)$ \\
\hline Sedentary & $0.48[0.23,0.98]$ & $0.720(0.045)$ & $0.79[0.50,1.25]$ & $1.000(0.318)$ \\
\hline
\end{tabular}

Data source: NCDS Study.

a $P$-values $(\alpha=0.05)$ were determined by logistic regression with Bonferroni correction for multiple comparisons.

${ }^{\mathrm{b}}$ Ordinal categories were: No formal education, Some primary education, Complete primary education, Complete secondary school or higher.

${ }^{c}$ No gender adjustment due to females having zero prevalence for the risk factor.

behavior in relation to emergence of NCD risk factors. The subgroups with higher purchasing capacity may become early adopters of lifestyles that promote NCD risk (31). Santiago may represent a community with mixed low- and middle-income families whose lifestyles produce a differential effect.
This study also revealed that adults in Santiago had a poor understanding of NCDs, with many respondents being unable to answer questions on relatively basic NCD-related concepts. For example, only one-fifth of individuals were able to correctly answer a multiple choice question that asked them to describe a classic heart attack. This suggests that basic cardiovascular health education is a potentially low-cost, easy-to-implement intervention through which to begin tackling NCDs.

Comparison between the reported prevalence of NCD risk factors in the Santiago population and rates in nonindigenous 
TABLE 5. Knowledge of noncommunicable diseases and their risk factors, as measured by number of questions answered correctly, among participants $(n=336)$ in the Noncommunicable Disease Surveillance (NCDS) study population in Santiago Atitlán, Guatemala, 2012-2013

\begin{tabular}{ccc}
\hline $\begin{array}{c}\text { Number of questions } \\
\text { answered correctly }\end{array}$ & $\begin{array}{c}\text { Percent answering correctly } \\
{[95 \% \mathrm{Cl}]}\end{array}$ & Cumulative percent \\
\hline / 9 & $0.7[0.0,1.6]$ & 0.7 \\
$1 / 9$ & $3.3[1.6,5.0]$ & 4.0 \\
2 / 9 & $7.5[4.0,11.0]$ & 11.6 \\
3 / 9 & $10.9[7.1,14.7]$ & 22.5 \\
$4 / 9$ & $19.8[14.3,25.4]$ & 42.3 \\
$5 / 9$ & $19.1[14.1,24.1]$ & 61.4 \\
$6 / 9$ & $18.5[12.8,24.3]$ & 79.9 \\
$7 / 9$ & $13.0[7.9,18.1]$ & 92.9 \\
$8 / 9$ & $4.6[1.4,7.7]$ & 97.5 \\
$9 / 9$ & $2.5[0.0,5.1]$ & 100.0 \\
\hline
\end{tabular}

Data source: NCDS Study.

Guatemalans and U.S. Latinos highlights that the indigenous population of Santiago Atitlán has NCD risk factor prevalences similar to communities further along in the epidemiologic transition. Indigenous communities in Guatemala share many cultural features and experience similar socioeconomic hardships and lack of adequate health care. Thus, it can be speculated that the findings of this study are likely generalizable to other indigenous communities in the country.

We found it challenging to work in a low-resource setting with major cultural barriers-a common hurdle in global health research. By applying a community-based, participatory approach that emphasized best practices and partnership with local organizations, we obtained a satisfactory response rate of $70 \%$. Furthermore, this approach enabled interviews to be conducted in the participants' native language, minimizing the risk of translation errors. Beyond the results of this study, bringing community groups together may have a lasting impact on NCD prevention efforts in the area. This approach contributed to local capacity building and helped establish connections for future collaboration.

1. Hunter DJ, Reddy KS. Noncommunicable diseases. New Engl J Med. 2013;369: 1336-43.

2. Gaziano TA, Bitton A, Anand S, AbrahamsGessel S, Murphy A. Growing epidemic of coronary heart disease in low- and middle-income countries. Curr Probl Cardiol. 2010;35(2):72-115.

\section{Limitations}

This population-based study utilized home-based surveys, limiting the sample to individuals at home during the day. The cultural role of women in the community and an unbalanced (i.e., lower) participation of women in the work force likely contributed to the over-representation of women in our sample (72.6\%). An attempt was made to correct for participation bias by applying multi-strata sampling weights to the analysis, but the risk of residual confounding or bias still exists. The $30 \%$ of screened individuals who did not respond and the additional $12 \%$ who did not complete biochemical measurements may have differed from those who participated, potentially introducing selection bias into the data. Thus, results must be interpreted with caution in the context of these limitations.

\section{Conclusions}

This community-based, participatory, cross-sectional study of noncommunicable disease risk factors in a rural Mayan community in a LMIC demonstrated that this community suffers from high rates of certain NCD risk factors. Specifically,

\section{REFERENCES}

3. Labarthe DR, Dunbar SB. Global cardiovascular health promotion and disease prevention: 2011 and beyond. Circulation. 2012;125:2667-76.

4. Barreto SM, Miranda JJ, Figueroa JP, Schmidt MI, Munoz S, Kuri-Morales PP, Silva JB. Epidemiology in Latin America and the Caribbean: current situation and high prevalences of obesity, hypertension, dyslipidemias, and metabolic syndrome were found, underscoring the need for effective, low-cost interventions to reduce the risk of noncommunicable diseases in this community and others like it.

It is likely that the high prevalence of risk factors will lead to rising rates of diabetes mellitus and cardiovascular disease in this community. It is important to confirm that similar trends are occurring in other indigenous populations in Guatemala to determine the generalizability of these findings for the purpose of applying common preventive efforts. In addition to actions aimed at improving health literacy relative to NCDs, systematic and cost-effective efforts for the detection of individuals with NCD risk factors are needed. Multi-institutional efforts to train health care personnel in NCD prevention and improve access to essential drugs would also be of great benefit in curtailing this emerging public health threat.

Acknowledgements. We are in debt to the community of Santiago Atitlán, Guatemala for allowing us to come into their community for this investigation. In particular, we would like to thank the community health workers and other local leaders that contributed to the success of this project: Leticia Ixcaya, Odilia Ixcaya, Marcos Sicay, Santiago Sicay, Ana Maritza Lacam, Josefa Sicay, Josefa Damián, Lucrecia Barán, and Marilu Quievac. We hope that this study contributes to the health of this community and others.

Conflicts of interest. The authors have no financial, personal, academic, or other conflicts of interest to declare.

Disclaimer. Authors hold sole responsibility for the views expressed in the manuscript, which may not necessarily reflect the opinion or policy of the RPSP/ PAJPH or PAHO. challenges. Int J Epidemiol. 2012;41(2): 557-71.

5. Roth GA, Forouzanfar MH, Moran AE, Barber R, Nguyen G, Feigin VL, Naghavi M, Mensah GA, Murray CJ. Demographic and epidemiologic drivers of global cardiovascular mortality. New Engl J Med. 2015;372:1333-41. 
6. Omran AR. The epidemiologic transition: a theory of the epidemiology of population change. Milbank Q. 2005;83(4): 731-57.

7. Ramirez-Zea M, Kroker-Lobos MF, CloseFernandez R, Kanter R. The double burden of malnutrition in indigenous and non-indigenous Guatemalan populations. Am J Clin Nutr. 2014;100(6):1644S-51S.

8. Garrett J, Ruel MT. The coexistence of child undernutrition and maternal overweight: prevalence, hypotheses, and programme and policy implications. Matern Child Nutr. 2005;1(3):185-96.

9. Ministry of Public Health and Social Assistance. National Epidemiological Bulletin No. 26. Guatemala City: Ministry of Public Health and Social Assistance; 2009.

10. Rozowski J, Castillo O, Moreno M, Bendich A. Effect of westernization of nutritional habits on obesity prevalence in Latin America. In: Bendich A, Deckelbaum RJ (eds). Preventive Nutrition: The Comprehensive Guide for Health Professionals, $3^{\text {rd }}$ ed. Totowa (NJ): Humana Press; 2005. Pp. 771-788.

11. Instituto Nacional de Estadística de Guatemala. Proyeccion Poblacion Año 2014. Guatemala City: Ministry of Public Health and Social Assistance; 2014.

12. Instituto Nacional de Estadística de Guatemala.Caracterización Departamental: Sololá 2012. Guatemala City: Ministry of Public Health and Social Assistance; 2013.

13. Schram J, Etzel N. Assessing community health in a Tz'tujil Maya village upon the reconstruction of Hospitalito Atitlán. Philadelphia: University of Pennsylvania; 2005.

14. Hernandez-Yol RA. Diagnóstico socioeconómico, potencialidades productivas $\mathrm{y}$ propuestas de inversión: Municipio de Santiago Atitlán, Departamento de Sololá. Guatemala City: Economics Faculty at the University of San Carlos; 2008.

15. World Health Organization. STEPwise approach to surveillance (STEPS) [internet]. Available from: http://www.who.int/ chp/steps/en/. Accessed May 2012.
16. Universidad de San Carlos. Distribución de la población por edad (censo). Guatemala City: University of San Carlos; 2005.

17. Verdich C, Barbe P, Petersen M, Grau K, Ward L, Macdonald I, et al. Changes in body composition during weight loss in obese subjects in the NUGENOB study: comparison of bioelectrical impedance vs. dual-energy X-ray absorptiometry. Diabetes Metab. 2011;37(3):222-9.

18. Flores R, Grajeda R, Torun B, Mendez H, Martorell R, Schroeder D. Evaluation of a dry chemistry method for blood lipid in field studies. FASEB J. 1998;12(Suppl): S3061.

19. World Health Organization. Physical status: the use and interpretation of anthropometry. Report of a WHO Expert Committee. World Health Organ Tech Rep Ser. 1995;854:1-452.

20. Gregory CO, Corvalan C, Ramirez-Z M, Martorell R, Stein AD. Detection of cardio-metabolic risk by BMI and waist circumference among a population of Guatemalan adults. Public Health Nutr. 2008;11(10):1037-45.

21. International Diabetes Federation (IDF). The IDF consensus worldwide definition of the metabolic syndrome [internet]. Brussels: IDF; 2006. Available at http:// www.idf.org/webdata/docs/IDF_Meta def_final.pdf. Accessed May 2012.

22. World Health Organization (WHO). WHO/ISH risk prediction charts for 14 WHO epidemiological sub-regions [internet]. WHO; 2007. Available at http://ishworld.com / downloads / activities / colour_charts_24_Aug_07.pdf. Accessed May 2012.

23. Sändal CE, Swensson B, Wretman J. Basic ideas in estimation from probability samples. In: Model Assisted Survey Sampling. New York: Springer-Verlag; 1992. Pp. 28-60.

24. Woodruff RS. A simple method for approximating the variance of a complicated estimate. J Am Stat Assoc. 1971;66: 411-4.

25. Iniciativa Centroamericana de Diabetes (CAMDI). Encuesta de diabetes, hipertensión y factores de riesgo de enfermedades crónicas: Villa Nueva, Guatemala, 2006. Washington, DC: Organización Panamericana de la Salud; 2007.

26. Daviglus ML, Talavera GA, Aviles-Santa ML, Allison M, Cai JW, Criqui MH, et al. Prevalence of major cardiovascular risk factors and cardiovascular diseases among Hispanic/Latino individuals of diverse backgrounds in the United States. JAMA. 2012;308(17):1775-84.

27. Arguelles W, Llabre MM, Sacco RL, Penedo FJ, Carnethon M, Gallo LC, et al. Characterization of metabolic syndrome among diverse Hispanics/ Latinos living in the United States: latent class analysis from the Hispanic Community Health Study/Study of Latinos (HCHS/SOL). Int J Cardiol. 2015;184:373-9.

28. Nagata JM, Valeggia CR, Barb FK, Bream KD. Body mass index, socio-economic status and socio-behavioral practices among Tz'utujil Maya women. Econ Hum Biol. 2009;7:96-106.

29. Cornier MA, Despres JP, Davis N, Grossniklaus DA, Klein S, Lamarche B, et al. Assessing adiposity: a scientific statement from the American Heart Association. Circulation. 2011;124(18):1996-2019.

30. World Health Organization Expert Consultation. Appropriate body-mass index for Asian populations and its implications for policy and intervention strategies. Lancet. 2004;363:157-63.

31. Murphy GA, Asiki G, Ekoru K, Nsubuga $\mathrm{RN}$, Nakiyingi-Miiro J, Young EH, et al. Sociodemographic distribution of noncommunicable disease risk factors in Uganda: a cross-sectional study. Int J Epidemiol. 2013;42:1740-53.

Manuscript received on 16 November 2015. Revised version accepted for publication on 4 March 2016. 
RESUMEN

\section{Prevalencia de los factores de riesgo de las enfermedades no transmisibles en una comunidad indígena de Santiago Atitlán, Guatemala}

Objetivo. Describir la prevalencia de los factores de riesgo de las enfermedades no transmisibles (ENT) y evaluar el conocimiento de esos factores de riesgo en la comunidad indígena de Santiago Atitlán en Guatemala, un país de ingresos medianos bajos. Métodos. Se realizó un estudio transversal basado en la población usando una versión modificada del protocolo STEPS de mediciones físicas de la Organización Mundial de la Salud. Se realizó una encuesta a adultos de 20 a 65 años de edad con respecto a algunas características demográficas y los factores de riesgo de las ENT; la encuesta fue seguida de mediciones antropométricas y bioquímicas.

Resultados. De las 501 personas encuestadas, se registraron 350 para el sondeo. La media de edad fue 36,7 años, y 72,3\% eran mujeres. Más de $90 \%$ informó tener un ingreso inferior a los US\$ 65 mensuales. Casi 80\% padecía retraso del crecimiento. Entre las mujeres, 37,3\% eran obesas y más de tres cuartas partes tenían obesidad central. Más de tres cuartas partes del grupo tenían dislipidemia y 18,3\% tenían hipertensión, pero solo 3,0\% tenía diabetes. En términos generales, 36,0\% de los participantes satisfacía los criterios de síndrome metabólico. No se observó una asociación significativa entre el nivel de escolaridad de los participantes y los factores de riesgo de las ENT, excepto una asociación inversa con la obesidad por porcentaje de tejidos grasos. Conclusiones. Santiago Atitlán es una comunidad indígena rural de Guatemala con tasas elevadas de pobreza y retraso del crecimiento, que coexisten con tasas altas de obesidad, en particular en las mujeres. Además, se encontraron tasas elevadas de hipertensión y dislipidemia, pero una tasa baja de diabetes mellitus. Se observó un conocimiento bajo de las ENT y sus factores de riesgo, lo que indica que las intervenciones educativas pueden ser un enfoque de alto rendimiento y bajo costo para combatir las ENT en esta comunidad.

Palabras clave Enfermedades cardiovasculares; enfermedades metabólicas; factores de riesgo; obesidad; población indígena; Guatemala. 